\title{
Exome sequencing and disease-network analysis of a single family implicate a mutation in KIF1A in hereditary spastic paraparesis
}

\author{
Yaniv Erlich, ${ }^{1,4,5}$ Simon Edvardson, ${ }^{2,4}$ Emily Hodges, ${ }^{3}$ Shamir Zenvirt, ${ }^{2}$ Pramod Thekkat, ${ }^{3}$ \\ Avraham Shaag, ${ }^{2}$ Talya Dor, ${ }^{2}$ Gregory J. Hannon, ${ }^{3}$ and Orly Elpeleg ${ }^{2}$ \\ ${ }^{1}$ Whitehead Institute for Biomedical Research, Cambridge, Massachusetts 02142, USA; ${ }^{2}$ Monique and Jacques Roboh Department \\ of Genetic Research, Department of Genetic and Metabolic Diseases, Hadassah, Hebrew University Medical Center, 91120 Jerusalem, \\ Israel; ${ }^{3}$ Watson School of Biological Sciences, Howard Hughes Medical Institute, Cold Spring Harbor Laboratory, Cold Spring Harbor, \\ New York 11724, USA
}

\begin{abstract}
Whole exome sequencing has become a pivotal methodology for rapid and cost-effective detection of pathogenic variations in Mendelian disorders. A major challenge of this approach is determining the causative mutation from a substantial number of bystander variations that do not play any role in the disease etiology. Current strategies to analyze variations have mainly relied on genetic and functional arguments such as mode of inheritance, conservation, and loss of function prediction. Here, we demonstrate that disease-network analysis provides an additional layer of information to stratify variations even in the presence of incomplete sequencing coverage, a known limitation of exome sequencing. We studied a case of Hereditary Spastic Paraparesis (HSP) in a single inbred Palestinian family. HSP is a group of neuropathological disorders that are characterized by abnormal gait and spasticity of the lower limbs. Forty-five loci have been associated with HSP and lesions in 20 genes have been documented to induce the disorder. We used whole exome sequencing and homozygosity mapping to create a list of possible candidates. After exhausting the genetic and functional arguments, we stratified the remaining candidates according to their similarity to the previously known disease genes. Our analysis implicated the causative mutation in the motor domain of KIFIA, a gene that has not yet associated with HSP, which functions in anterograde axonal transportation. Our strategy can be useful for a large class of disorders that are characterized by locus heterogeneity, particularly when studying disorders in single families.
\end{abstract}

[Supplemental material is available for this article. The sequencing and genotyping data from this study have been submitted to the NCBI dbGaP (http:// www.ncbi.nlm.nih.gov/gap) and at http:// cancan.cshl.edu/hsp/.]

Whole exome sequencing has ushered in a renaissance in identifying pathogenic variations in monogenic diseases. The approach enables a rapid and cost-effective detection from a small number of individuals, and has proved useful for a wide range of clinical settings (Choi et al. 2009; Ng et al. 2009, 2010a, 2010b; Krawitz et al. 2010; Pierce et al. 2010; Walsh et al. 2010). A major challenge of whole exome sequencing is determining the causative mutation from a substantial number of bystander variations that do not play a role in the disease etiology. The common strategy to filter out the bystander variations is based on a systematic rejection of variations according to genetic and functional arguments, narrowing down the candidate list until the causative variation is isolated. One class of genetic arguments that has been widely used is rejection of variations that are not shared between multiple cases or that do not follow the assumed mode of inheritance ( $\mathrm{Ng}$ et al. 2009, 2010b; Krawitz et al. 2010). Another class of genetic arguments asserts that harmful mutations must be rare due to purifying selection and accordingly reject variations that have been catalogued in dbSNP or 1000 Genomes, as these are assumed to be relatively common. Functional arguments focus on the impact of the variation on the

\footnotetext{
${ }^{4}$ These authors contributed equally to this work.

${ }^{5}$ Corresponding author.

E-mail yaniv@wi.mit.edu.

Article published online before print. Article, supplemental material, and publication date are at http://www.genome.org/cgi/doi/10.1101/gr.117143.110.
}

protein, either by analyzing biochemical and structural features (Adzhubei et al. 2010) or by measuring the extent of multispecies conservation at the variation site (Cooper et al. 2010).

Monogenic disorders in isolated inbred families cast a unique setting for identifying the causative variations. Homozygosity mapping can quickly identify regions that are identical by decent, refining the search area to regions that typically span several megabases (Lander and Botstein 1987). However, this setting brings several challenges. First, bystander variations inside the homozygous region are (almost) always homozygous; therefore, they are not amenable for rejection based on mode of inheritance (Choi et al. 2009; Walsh et al. 2010). Second, the disease-harboring region is identical between the affected siblings and sequencing multiple cases from the same family adds minimal information beyond the data from the homozygosity mapping. Third, inbred unions are more prevalent in non-Western societies, such as North Africa, the Middle East, and Central Asia (Bittles 2001). These ethnic groups tend to be less represented in variation repositories (Carlson et al. 2003; Via et al. 2010), reducing the power of eliminating common polymorphisms.

In this study, we present disease-network analysis as an additional layer of arguments to stratify variations. Accumulating lines of evidence have shown that genes that are associated with phenotypically close disorders are prone to have similar molecular signatures (Goh et al. 2007; Feldman et al. 2008; Wu et al. 2008). These include similar expression profiles, participation in the same 
signaling or metabolic pathways, or sharing similar protein domains. Thus, it is appealing to reject variations by comparing the molecular signature of their harboring genes to the signatures of closely related disease genes.

Unlike most of the rejection arguments above, disease-based arguments do not require sequencing information per se. This provides a means to exclude positions that were not covered or miscalled, a common caveat in whole exome sequencing ( $\mathrm{Ng}$ et al. 2010a). Disease-network analysis has been mainly used for gene prioritizing as the first step of gene-centric studies, when sequencing was a limiting factor (Ropers 2007). One drawback of this approach is the requirement to prioritize a large number of genes, which increases the amount of false positives. In our approach, the analysis is executed at the last stage on a minimal list of genes, increasing the specificity of the method.

\section{Results}

\section{Description of the affected family}

We evaluated the performance of our strategy in a case of three brothers aged 20 (Patient II-4), 15 (Patient II-5), and 14 (Patient II-7) years, who presented to our clinic with a chief complaint of abnormal gait. The parents were of Muslim Palestinian origin and denied consanguinity; however, both originated from the same village. They and the other children were reportedly healthy at 16 to $23 \mathrm{yr}$ old (Fig. 1). The perinatal course and early development of all patients were uneventful and walking appeared at around one year of age. Stiff legs and a slowly progressive gait disturbance became evident at two years, but both participated successfully in sport activities throughout childhood. Further aggravation of symptoms was noted at 10-13 yr of age and the patients could no longer play soccer or walk long distances. Lower limb sensation, sphincters, upper limbs, and intellectual functions were intact throughout childhood.

Patient II-4, when first examined at age 20, was unable to run. His gait was stiff with equinos-gait, scissoring, and crouching. He had increased reflexes in his lower limbs, plantar reflexes were extensor, and bilateral ankle clonus was elicited. Bilateral spasticity on ankle dorsiflexion, hip adduction, and knee extension was 3 according to the modified Ashworth scale. The rest of the physical examination was unremarkable. Patient II-5, a 15-yr-old male, was noted to have difficulty in running. The tendon reflexes were increased in his lower limbs and the plantar reflexes were extensor but ankle clonus was not elicited. Spasticity was noted on ankle dorsiflexion and hip adduction up to a modified Ashworth score of 2. The rest of the physical examination was normal. Patient II-7, at $14 \mathrm{yr}$, was able to run slowly, with a tendency to tiptoeing. Physical examination disclosed increased reflexes in the lower limbs, ankle clonus, and extensor plantar responses. Reflexes were normal in the upper limbs. Spasticity was evident in both lower limbs at rest with a modified Ashworth score of 3 on ankle dorsiflexion. Knee flexion and hip adduction were less affected with an Ashworth

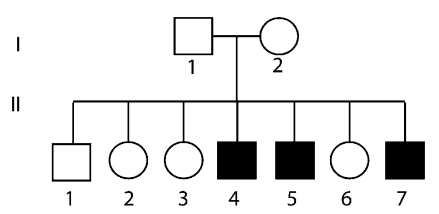

Figure 1. Pedigree of the affected family. Sex of the nonaffected individuals was randomized to prevent identification of the family. score of 2. No weakness, wasting, decreased vibration sense, or pinprick sensation was noted, and there were no sphincter disturbances or cerebellar signs. Brain MRI in all patients was normal. EMG and NCV in II-4 and II-5 were normal.

Based on these results, the patients were diagnosed with a pure type of Hereditary Spastic Paraparesis (HSP). HSP is a group of genetic disorders resulting in axonal degradation of the corticospinal tract (Reid 2003; Dion et al. 2009). They are characterized by a progressive lower-extremity spastic weakness, hypertonic urinary bladder disturbance, mild diminution of lower-extremity vibration sensation and, occasionally, of joint position sensation. As of September 2010, 45 HSP loci and 20 HSP-related genes have been identified (Supplemental Table 1), showing autosomal dominant, autosomal recessive, and X-linked inheritance patterns (Dion et al. 2009). HSPs are subdivided into pure and complicated forms, where the complicated forms involve additional pathologies, such as mental retardation, ocular signs, and skin abnormalities. However, some loci are associated with both pure and complicated forms of HSPs (Reid 2003; Dion et al. 2009).

\section{Rejecting involvement of known HSP genes or X-linked disorders}

We determined the complete exonic sequences of patient II-5 and his parents, using array-based hybrid selection and Illumina sequencing (see Methods), and we genotyped the whole genome of Patient II-4 and Patient II-5, using GeneChip Human Mapping 250K Nsp Array of Affymetrix, as previously described (Edvardson et al. 2007).

We first evaluated the possibility that the disorder is caused by known HSP genes. Patient II-4 and II-5 shared 610 Mbase (23\%) of identical autosomal segments, and 75 Mbase (50\%) of identical segments on the $\mathrm{X}$ chromosome, as expected from full siblings. The shared autosomal segments contained four known HSP genes: ATL1, GJC2, HSPD1, and SPG20. The sequencing coverage was 99\% for the coding regions of these genes. We could not find any homozygous variation or compound heterozygous variations in the genes that can explain the disorder (Supplemental data and Supplemental Table 2). In addition, a search for an X-linked mutation did not find any potential variation (Supplemental data and Supplemental Table 3).

We also compared a list of homozygous SNPs from the entire autosome of patient II-5 to more than 40,000 known pathogenic variations in the Human Gene Mutation Database (HGMD) (Stenson et al. 2003) revision 2009.2. This did not identify any previously known variation that can account for the observed phenotype. Our systematic analysis indicates that the disorder is caused by a new autosomal gene.

\section{Analyzing homozygous regions}

Homozygous runs have an overwhelming probability to carry disease mutations in inbred families (Lander and Botstein 1987). The array data revealed four homozygous runs that are shared between the brothers and that are longer than 1 Mbase. The longest segment spanned $>2.5 \mathrm{Mb}$ at the tip of chromosome 2 (Table 1 ). The length of homozygous runs is inversely correlated with the number of meiosis events from the common ancestor (Clark 1999). Therefore, the longest segment is prone to hold the disease mutation with a probability that is nonproportional to its relative length (Woods et al. 2006). In order to maximize the sensitivity of our analysis, we included all four homozygous runs, with the expectation that the longest region will harbor the disease mutation. 
Table 1. Homozygous regions $>1000 \mathrm{~K}$ that are shared between II-4 and II-5

\begin{tabular}{lcccccr}
\hline Chr. & Start & Stop & $\begin{array}{c}\text { Homo. } \\
\text { SNPs }\end{array}$ & Size & $\begin{array}{c}\text { Number of } \\
\text { genes }\end{array}$ & $\begin{array}{r}\text { Coding } \\
\text { positions }\end{array}$ \\
\hline 2 & $240,066,688$ & $242,650,580$ & 132 & $2584 \mathrm{~K}$ & 36 & 61,483 \\
3 & $103,303,758$ & $104,720,622$ & 125 & $1417 \mathrm{~K}$ & 1 & 1,373 \\
2 & $234,755,156$ & $235,930,278$ & 111 & $1175 \mathrm{~K}$ & 2 & 3,506 \\
10 & $100,073,394$ & $101,203,270$ & 119 & $1129 \mathrm{~K}$ & 5 & 10,226 \\
& & & Total & $6306 \mathrm{~K}$ & 44 & 76,588 \\
\hline
\end{tabular}

The four homozygous runs contained 44 genes that consist of 76,588 bases that are either translated or reside in splicing sites. The sequencing data of patient II-5, the mother, and the father covered $90 \%, 90 \%$, and $89 \%$ of these bases, respectively. Importantly, when we examined the $7.8 \mathrm{~K}$ bases that were not covered, we found that $5.6 \mathrm{~K}$ bases were part of exons that were not included in the design of the capture array. All of these exons reside in the longest homozygous run on the tip of chromosome 2 . We did not exclude the positions that were not covered by sequencing, and we treated them as hypothetical suspects until proven innocent.

We applied a series of exclusion steps to uncover the causative mutation: (1) We rejected positions that matched the reference, reducing the candidate list to 8083 positions, out of which 213 positions were variants detected using the sequencing data; (2) by analyzing the sequencing data from the parents, we excluded positions that were homozygous in one of the two parents; (3) variations that were documented in dbSNP130 or in the 1000 Genomes project were excluded; (4) we rejected synonymous substitutions; (5) we removed positions that were not subject to even a modest purifying selection, as indicated by a score of zero or less in GERP (Cooper et al. 2005). Five thousand ninety-eight positions in 15 genes passed this series of exclusion criteria, all of which reside in the longest homozygous run (Table 2). However, only five positions were variations that were called according to the sequencing information, whereas the rest of the candidate positions were unknowns, mostly due to the absence of a corresponding probe in the array design.

We turned to disease-network analysis to identify the pathogenic variation. We composed a list of all the known genes that are associated with a pure type of HSP (Table 3), and measured their signature similarity to the 15 candidate genes. To increase the robustness of our analysis, we used three algorithms: SUSPECTS (Supplemental Table 4; Adie et al. 2005), ToppGene (Supplemental Table 5; Chen et al. 2007), and Endeavour (Supplemental Table 6; Aerts et al. 2006). Each of these algorithms relies on a distinct combination of features and metrics to characterize the signature of similar disease-causing genes. SUSPECTS mainly uses sequence features, such as GC content and gene length; ToppGene integrates mouse phenotype data; and Endeavour fuses multiple data sources, including gene ontology, text mining, and expression data. The results were univocal: KIF1A was the top candidate in all algorithms (Table 4). This was supported by gene interaction, motif score, annotation similarity, sequence similarity to other disease genes, and mouse phenotype data.

We repeated the Endeavour analysis with a training set that did not include KIF5A in order to circumvent possible in KIF1A. positions that were covered in patient II-5. biases due to high similarity to KIF1A. Again, KIF1A was the top gene. To check the robustness of the results, we randomly selected subsets of five training genes and repeated the Endeavour analysis for the entire candidate list. In nine out of 10 times KIF1A was the top gene. Finally, we trained Endeavour with the entire set of 20 genes that cause either a complicated or pure form of HSP. KIF1A was also the top gene in this setting.

KIF1A had complete sequencing information, and the only variation that passed the multiple exclusion criteria was Ala255Val. As a complementary approach, we performed a loss of function analysis on the five variations that passed the elimination process. We used MutationTaster (Schwarz et al. 2010), Polyphen (Adzhubei et al. 2010), and SIFT (Ng and Henikoff 2003) to classify the variations. All of these analysis methods implicated Ala225Val in KIF1A as a harmful mutation. The results on the other variations were not consistent between the tools, except a variation in HDLBP that was also found harmful (Fig. 2). Using Sanger sequencing, we found that the putative variation in $H D L B P$ was a sequencing error (Supplemental Fig. 1). Therefore, the only harmful variation according to our stringent analysis is Ala255Val

We validated the KIF1A mutation by Sanger sequencing, confirming that the three patients are homozygous to this variation, whereas the parents and four unaffected siblings were heterozygous (Supplemental Fig. 2). There is less than $0.5 \%$ chance of this segregation pattern in the children at random. To determine the carrier rate, we genotyped 573 anonymous individuals of the same ethnic origin and found three carriers, indicating a carrier rate of 1:191 in this population (95\% confidence interval: $0.06 \%-$ $1.15 \%)$, as expected from a rare disease caused by a relatively ancient founder in a genetic isolate.

The autosomal-recessive HSP30 (MIM 610357) had been previously linked to a $5.1 \mathrm{cM}$ interval on chromosome $2 \mathrm{q} 37.3$ that encompasses the KIF1A gene (Klebe et al. 2006). The maximum multipoint LOD score was between markers D2S2338 and D2S2585 (chr2: 238,514,684-242,575,273). The four reported HSP30 patients presented in adolescence with spastic gait, distal wasting, sensory neuropathy, and cerebellar ataxia with mild diffuse cerebellar atrophy. In our patients, the disease presented in infancy and has only involved spastic gait without additional abnormalities. As noted above, it has been shown that different lesions in the same HSP associated genes can create a range of phenotypes and account for both pure and complicated forms of

Table 2. A description of the rejection process of the positions in the homozygous regions

\begin{tabular}{|c|c|c|c|c|}
\hline \multirow{2}{*}{$\begin{array}{l}\text { Exclusion method } \\
\text { Candidates }\end{array}$} & \multicolumn{2}{|c|}{ Total genes positions } & \multicolumn{2}{|c|}{$\begin{array}{l}\text { Sequenced genes } \\
\text { positions }\end{array}$} \\
\hline & 44 & 76588 & 41 & 68718 \\
\hline ... not homozygous WT & 40 & 8083 & 35 & 213 \\
\hline ... AND parental is not homozygous & 24 & 7232 & 17 & 39 \\
\hline ... AND not in dbSNP/1000 Genomes & 18 & 7184 & 7 & 12 \\
\hline ... AND not synonymous changes & 17 & 7028 & 6 & 8 \\
\hline$\ldots$ AND conserved & 15 & 5098 & 5 & 5 \\
\hline ... disease-network analysis & 1 (KIF1A) & 1 (KIF1A) & & - \\
\hline$\ldots$ loss of function analysis & - & - & 1 (KIF1A) & 1 (KIF1A) \\
\hline
\end{tabular}

Total: all possible positions, including positions without sequencing information. Sequenced: only 
Table 3. A list of genes that are known to be associated with pure types of HSPs and used to obtain the disease signature

\begin{tabular}{lll}
\hline Gene name & OMIM & \multicolumn{1}{c}{ Phenotype } \\
\hline CYP7B1 & 603711 & SPG5, Bile acid synthesis defect \\
HSPD1 & 118190 & SPG13, hypomelinating leukodystrophy \\
KIAA0196 & 610657 & SPG8 \\
KIF5A & 602821 & SPG10 \\
NIPA1 & 608145 & SPG6 \\
PLP1 & 300401 & SPG2, Pelizaeus-Merzbacher disease \\
PNPLA6 & 603197 & SPG39 \\
REEP1 & 609139 & SPG31 \\
SPAST & 604277 & SPG4 \\
ATL1 & 606439 & SPG3A \\
ZFYVE27 & 610243 & SPG33 \\
\hline
\end{tabular}

HSP. Therefore, our data could suggest that KIF1A accounts for HSP30.

\section{Characterization of KIFIA}

KIF1A consists of 48 exons that encode a 1791-amino-acid kinesin (Fig. 3A). Residues 1-367 of the protein form the motor domain and Ala 255 is part of a nine amino acids stretch that is fully conserved throughout fungi, nematode, insect, and vertebrates (Fig. 3C). It is adjacent to the tip of loop L11 (Kull et al. 1996), a key structural element in the catalytic core of the protein (Hirokawa et al. 2009). Figure 3B presents the location of Ala 255 on a 3D model of KIF1A motor domain based on the PDB entry 1VFZ (Nitta et al. 2004).

\section{Discussion}

We used genetic, functional, and disease-network analysis arguments to associate a mutation in a novel gene, KIF1A, with a pure form of HSP cases in a single inbred family. Our combined approach enabled us to examine all of the translated positions in the genome, including positions that were not covered due to incomplete design of the capture array, and to systematically reject all but one position in KIF1A.

Kinesins are a large superfamily of molecular motors; they use microtubules as a "rail" to transport cargo along, and chemical energy of ATP to drive conformational changes that generate motile force. Active transport of proteins along directional cytoskeletal filaments to their appropriate destination using molecular motors is most prominent in polarized cells, including neurons, and is fundamental for neuronal function and survival because most of the proteins required in the axon and nerve terminals need to be transported from the cell body. There are 45 mammalian KIF genes and the encoded proteins are classified into 15 kinesin families, based on phylogenetic analyses; KIF1A belongs to the kinesin 3 family. The 15 families are grouped according to the position of the motor domain in the molecule. Hence, KIF1A is an N-kinesin, as its motor domain, which associates with microtubules, is in the aminoterminal region. Like other N-kinesins, KIF1A drives the microtubule plus end toward the axon terminal, thus powering anterograde transport. At the axon terminus, neurotransmitters containing synaptic vesicles are produced by endocytosis. These vesicles contain proteins that have been transported to the plasma membrane in synaptic vesicle precursors. The kinesin
3 family motors KIF1A and KIF1B (beta isoform) transport synaptic vesicle precursors that contain the synaptic vesicle proteins, synaptophysin, synaptotagmin, and the small GTPase RAB3A (Hall and Hedgecock 1991; Okada et al. 1995; Hirokawa et al. 2009). At the axon terminal, RAB3 controls the exocytosis of synaptic vesicles.

Kinesins have long been implicated in the pathogenesis of axonal neuropathies. A loss-of-function mutation in the ATP binding consensus of $K I F 1 B$ motor domain was associated with Charcot-Marie-Tooth neuropathy type 2A (Zhao et al. 2001). Mutations in the motor domain of KIF5A account for SPG1038 and heterozygous missense mutations in KIF21A are the cause of congenital fibrosis of the extraocular muscle type 1 (Yamada et al. 2003). A mutation in unc-104, the C. elegans ortholog of $K I F 1 A$, is associated with decreased transport of synaptic vesicle precursors in the axons (Otsuka et al. 1991). Knockout of Kif1a in mice is lethal soon after birth due to massive axonal and neuronal cell body degeneration at the central nervous system, accompanied by motor and sensory disturbances, more pronounced in the hindlimbs. Transport of synaptic vesicle precursor proteins, not only those carried by Kif1a, is decreased. This is evident by the abnormal clustering of vesicles in the cell bodies of the neurons, their reduced accumulation in ligation experiments, and the reduced number of synaptic vesicles. This finding was unexpected, as the transport of synaptic plasma membrane precursors seems intact. Nonetheless, the reduction in synaptic vesicles was associated with a decrease in nerve terminal number (Yonekawa et al. 1998). Knockout of the Kif1b gene in mice had a similar phenotype, with lethality in the perinatal period and severe neuronal degeneration and synaptic dysfunction (Zhao et al. 2001). Mice that were heterozygous for the Kif1b gene are viable, but suffer from progressive peripheral neuropathy. These studies and the present report, suggest that although redundantly transporting the same cargoes, Kif1 $a$ and Kif1b cannot compensate for each other; an abnormally low level of either one of them results in neuronal phenotype.

Disease-network analysis adds a complementary layer of information to the sequencing data. It provides a means to integrate prior knowledge on the expected molecular signature of the disease from closely associated conditions and to reject

Table 4. The rank of the candidate genes in different disease prediction algorithms and the combined results

\begin{tabular}{lcccr}
\hline & \multicolumn{5}{c}{ Rank } \\
\cline { 2 - 5 } Gene & SUSPECTS & ToppGene & Endeavour & Combined \\
\hline KIF1A & $\mathbf{1}$ & $\mathbf{1}$ & $\mathbf{1}$ & $\mathbf{1}$ \\
D2HGDH & 2 & 2 & 3 & 2 \\
ATG4B & 5 & 3 & 5 & 3 \\
HDLBP & 3 & 11 & 4 & $4-5$ \\
PASK & 9 & 7 & 2 & 6 \\
ING5 & 4 & 8 & 8 & 7 \\
SNED1 & 12 & 5 & 6 & 8 \\
DTYMK & 10 & 9 & 7 & \\
OR6B2 & 11 & 10 & 9 & $9-12$ \\
OR6B3 & 13 & 6 & 11 & \\
AQP12B & 7 & 12 & 13 & $13-14$ \\
AQP12A & 6 & 13 & 14 & 15 \\
LOC728846 & 14 & 4 & 15 & \\
THAP4 & 8 & 14 & 12 & \\
LOC643905 & 15 & 15 & 10 & \\
\hline
\end{tabular}

KIF1A was scored as the top candidate in all three prediction algorithms. 


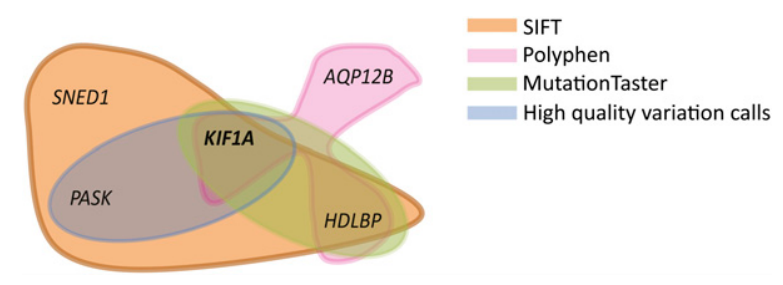

Figure 2. Loss of function prediction: KIF1A scores in all the tools. (Orange) The subset of variations that were predicted as harmful by SIFT. (Pink) Harmful variations by PolyPhen. (Green) Harmful variations by MutationTaster. (Blue) High-quality variations based on SNVmix scores. The HDBLP variation was later proved to be a sequencing error. KIF1A is the only harmful variation.

bystander variations. The disease-network analysis approach has been mainly used to prioritize gene lists as the first step of candidate gene studies. In this study, we used gene prioritization as the final step, after exhausting genetic arguments. We found that the analysis is robust across different algorithms and random subsets of training disease genes.

The OMIM database describes more than 200 Mendelian conditions that show locus heterogeneity with a mixture of known and unknown loci. Our combined approach provides a rich layer of information for medical sequencing of those conditions.

\section{Methods}

\section{Human samples}

All experiments involving DNA of the patients and their relatives were approved by the Hadassah Ethical Review Committee.

\section{Illumina sequencing}

Blood derived DNA samples were fragmented and immortalized by ligation to standard Illumina adapters, followed by PCR-based enrichment (Hodges et al. 2009). Sequences corresponding to human exons were enriched by hybridization to an Agilent 1 Million Feature Array (Ng et al. 2009) and sequenced using the 76 paired-end standard Illumina kit.

We used Bowtie (Langmead et al. 2009) to align the reads to the human genome reference version NCBI36/hg18. We employed the following strategy to align the reads: align pair-end reads allowing up to two mismatches and suppress multiple mappers; pair-end reads that failed to align in the previous round were broken to single-end reads and realigned again allowing up to two mismatches and no multiple mappers; reads that failed to align in the previous round were trimmed by $10 \mathrm{bp}$ and realigned again. We repeated the trimming and realignment up to a length of $36 \mathrm{nt}$, while suppressing multiple

\section{A}

B

C mappers in every step. In total, we accumulated 3.6 Gbase of sequencing information for the affected child, 4.8 Gbase for the mother, and 2.2 Gbase for the father. The average fold coverage of patient II-5 was 31 and the median was 25 for autosomal coding regions as defined in RefSeq hg18; the average fold coverage of the mother was 57 and the median was 49 ; the average fold coverage of the father was 20 and the median was 17 (Supplemental Fig. 3).

We called SNPs with SNVmix (Goya et al. 2010) using the default parameters and we instructed the program to also report positions without variations using a small change in the source code (available by request from the authors). Reads that failed to align with Bowtie were realigned with BWA (Li and Durbin 2009) allowing up to three indels that are not $5 \mathrm{bp}$ from the end of the read (BWA aln i5 n3). We used VarScan (Koboldt et al. 2009) to call indels using the following command parameters: --mincoverage 3 --min-var-freq 0.3 --min-reads2. Homozygous indels were called only when $90 \%$ of the sequence reads reported the nonreference allele.

By comparing the genotyping results of the array to the sequencing data of patient II-5, we estimate that the sensitivity of calling nonreference homozygous SNPs was 99\% and 94\% for
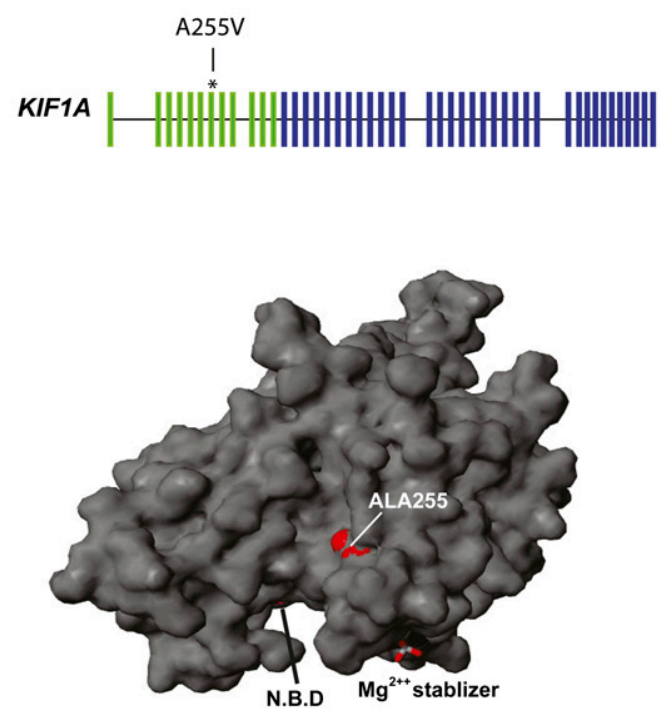

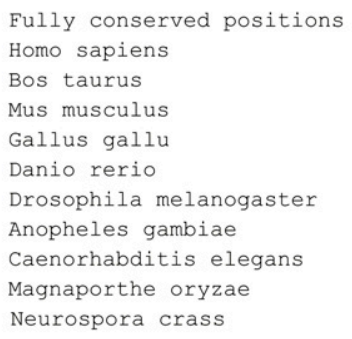

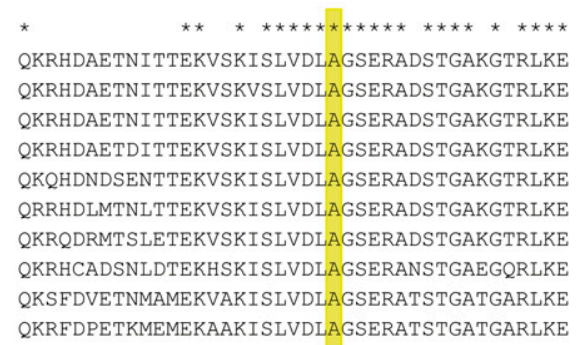

Figure 3. Ala255Val is a mutation in the protein motor area of KIF1A. (A) Schematic representation of the KIF1A gene, the exons that encode the motor domain (green), and the location of the mutation. $(B)$ A 3D model of KIF1A motor domain. Ala 255 is highlighted. The nucleotide binding pocket (NBP) and the magnesium stabilizer are found in close proximity to the mutation. (C) The amino acid sequence of KIF1A homologs in the vicinity of Ala255 (yellow). Positions that are labeled with a star are fully conserved between human to fungi.

\section{Genome Research}


heterozygous SNPs. The false discovery rate was $1.8 \%$ for homozygous SNPs and $1.4 \%$ for heterozygous SNPs.

\section{Finding identical segments and homozygous regions using genotype arrays}

In order to find identical segments, we compared the genotyping results of the two brothers using a sliding window of 100 SNPs $(\sim 1 \mathrm{cM})$, allowing up to $3 \%$ errors. Overlapping windows that passed the threshold were merged into one segment. We used plink (Purcell et al. 2007) to identify homozygous SNPs using the following parameters: --homozyg --homozyg-group --homozygwindow-het 0 . To enhance the accuracy, we excluded discordant SNPs in the identical segments from the input ped file.

\section{Exclusion process}

Positions that were homozygous in the parents (wild-type or mutated) were excluded from the analysis. In order to increase the sensitivity, we used parental data to extrapolate about 450 positions that were called as heterozygous in one of the parents and were not covered in the patient. In all those cases, we assumed a worst case scenario, and treated them as homozygous variations in the patient. All of these variations were excluded based on other criteria.

SeattleSeq Annotation (http://gvs.gs.washington.edu/ SeattleSeqAnnotation/HelpAbout.jsp) was used to analyze the variations. Endeavour (http://homes.esat.kuleuven.be/ bioiuser/ endeavour/tool/endeavourweb.php) was trained with all possible features except BLAST. ToppGene (http://toppgene.cchmc.org/ prioritization.jsp) and SUSPECTS (http://www.genetics.med.ed. ac.uk/suspects/) were used with the default training parameters.

\section{Carrier rate determination}

We employed the TaqMan Allelic Discrimination method with the forward primer 5'-AAATCAGCCTGGTGGACCTG-3', reverse primer 5'-CCTGGCCCCTACCTTGAG-3', and the following reporter probes: wild-type, VIC probe 5'-TGGAGTCAGCCCGCTC-3'; mutant, FAM probe 5'-TGGAGTCAACCCGCTC-3'. The analysis was performed on a 7900HT Real-Time PCR System with SDS version 2.3 software (Applied Biosystems). With this method, heterozygosity for the mutation was detected in three of 573 anonymous individuals of the same ethnic origin.

\section{Access to the sequencing and genotyping data sets}

The sequencing and genotyping data from this study are available at dbGAP (http://www.ncbi.nlm.nih.gov/gap) and at http:// cancan.cshl.edu/hsp/ (please refer to the website for the Terms and Conditions).

\section{Acknowledgments}

We thank Melissa Gymrek for useful comments. This work was supported by a kind gift from Kathryn W. Davis and by the Joint Research Fund of the Hebrew University and Hadassah Medical Organization. Y.E. is an Andrea and Paul Heafy Family Fellow of the Whitehead Institute for Biomedical Research. G.J.H. is an investigator of the Howard Hughes Medical Institute.

\section{References}

Adie EA, Adams RR, Evans KL, Porteous DJ, Pickard BS. 2005. Speeding disease gene discovery by sequence based candidate prioritization. BMC Bioinformatics 6: 55. doi: 10.1186/1471-2105-6-55.
Adzhubei IA, Schmidt S, Peshkin L, Ramensky VE, Gerasimova A, Bork P, Kondrashov AS, Sunyaev SR. 2010. A method and server for predicting damaging missense mutations. Nat Methods 7: 248-249.

Aerts S, Lambrechts D, Maity S, Van Loo P, Coessens B, De Smet F, Tranchevent LC, De Moor B, Marynen P, Hassan B, et al. 2006. Gene prioritization through genomic data fusion. Nat Biotechnol 24: 537-544.

Bittles A. 2001. Consanguinity and its relevance to clinical genetics. Clin Genet 60: 89-98.

Carlson CS, Eberle MA, Rieder MJ, Smith JD, Kruglyak L, Nickerson DA. 2003. Additional SNPs and linkage-disequilibrium analyses are necessary for whole-genome association studies in humans. Nat Genet 33: 518-521.

Chen J, Xu H, Aronow BJ, Jegga AG. 2007. Improved human disease candidate gene prioritization using mouse phenotype. $B M C$ Bioinformatics 8: 392. doi: 10.1186/1471-2105-8-392.

Choi M, Scholl UI, Ji W, Liu T, Tikhonova IR, Zumbo P, Nayir A, Bakkaloglu A, Ozen S, Sanjad S, et al. 2009. Genetic diagnosis by whole exome capture and massively parallel DNA sequencing. Proc Natl Acad Sci 106: 19096-19101.

Clark AG. 1999. The size distribution of homozygous segments in the human genome. Am J Hum Genet 65: 1489-1492.

Cooper GM, Stone EA, Asimenos G, Green ED, Batzoglou S, Sidow A. 2005. Distribution and intensity of constraint in mammalian genomic sequence. Genome Res 15: 901-913.

Cooper GM, Goode DL, Ng SB, Sidow A, Bamshad MJ, Shendure J, Nickerson DA. 2010. Single-nucleotide evolutionary constraint scores highlight disease-causing mutations. Nat Methods 7: 250-251.

Dion PA, Daoud H, Rouleau GA. 2009. Genetics of motor neuron disorders: new insights into pathogenic mechanisms. Nat Rev Genet 10: 769-782.

Edvardson S, Shaag A, Kolesnikova O, Gomori JM, Tarassov I, Einbinder T, Saada A, Elpeleg O. 2007. Deleterious mutation in the mitochondrial arginyl-transfer RNA synthetase gene is associated with pontocerebellar hypoplasia. Am J Hum Genet 81: 857-862.

Feldman I, Rzhetsky A, Vitkup D. 2008. Network properties of genes harboring inherited disease mutations. Proc Natl Acad Sci 105: 4323-4328.

Goh KI, Cusick ME, Valle D, Childs B, Vidal M, Barabasi AL. 2007. The human disease network. Proc Natl Acad Sci 104: 8685-8690.

Goya R, Sun MG, Morin RD, Leung G, Ha G, Wiegand KC, Senz J, Crisan A, Marra MA, Hirst M, et al. 2010. SNVMix: predicting single nucleotide variants from next-generation sequencing of tumors. Bioinformatics 26: 730-736.

Hall DH, Hedgecock EM. 1991. Kinesin-related gene unc-104 is required for axonal transport of synaptic vesicles in C. elegans. Cell 65: 837-847.

Hirokawa N, Nitta R, Okada Y. 2009. The mechanisms of kinesin motor motility: lessons from the monomeric motor KIF1A. Nat Rev Mol Cell Biol 10: $877-884$.

Hodges E, Rooks M, Xuan Z, Bhattacharjee A, Benjamin Gordon D, Brizuela L, Richard McCombie W, Hannon GJ. 2009. Hybrid selection of discrete genomic intervals on custom-designed microarrays for massively parallel sequencing. Nat Protoc 4: 960-974.

Klebe S, Azzedine H, Durr A, Bastien P, Bouslam N, Elleuch N, Forlani S, Charon C, Koenig M, Melki J, et al. 2006. Autosomal recessive spastic paraplegia (SPG30) with mild ataxia and sensory neuropathy maps to chromosome 2q37.3. Brain 129: 1456-1462.

Koboldt DC, Chen K, Wylie T, Larson DE, McLellan MD, Mardis ER, Weinstock GM, Wilson RK, Ding L. 2009. VarScan: variant detection in massively parallel sequencing of individual and pooled samples. Bioinformatics 25: 2283-2285.

Krawitz PM, Schweiger MR, Rodelsperger C, Marcelis C, Kolsch U, Meisel C, Stephani F, Kinoshita T, Murakami Y, Bauer S et al. 2010. Identity-bydescent filtering of exome sequence data identifies PIGV mutations in hyperphosphatasia mental retardation syndrome. Nat Genet 42: 827-829.

Kull FJ, Sablin EP, Lau R, Fletterick RJ, Vale RD. 1996. Crystal structure of the kinesin motor domain reveals a structural similarity to myosin. Nature 380: $550-555$.

Lander ES, Botstein D. 1987. Homozygosity mapping: a way to map human recessive traits with the DNA of inbred children. Science 236: 1567-1570.

Langmead B, Trapnell C, Pop M, Salzberg SL. 2009. Ultrafast and memoryefficient alignment of short DNA sequences to the human genome. Genome Biol 10: R25. doi: 10.1186/gb-2009-10-3-r25.

Li H, Durbin R. 2009. Fast and accurate short read alignment with BurrowsWheeler transform. Bioinformatics 25: 1754-1760.

$\mathrm{Ng}$ PC, Henikoff S. 2003. SIFT: Predicting amino acid changes that affect protein function. Nucleic Acids Res 31: 3812-3814.

Ng SB, Turner EH, Robertson PD, Flygare SD, Bigham AW, Lee C, Shaffer T, Wong M, Bhattacharjee A, Eichler EE, et al. 2009. Targeted capture and massively parallel sequencing of 12 human exomes. Nature 461: 272-276.

$\mathrm{Ng} \mathrm{SB}$, Bigham AW, Buckingham KJ, Hannibal MC, McMillin MJ, Gildersleeve HI, Beck AE, Tabor HK, Cooper GM, Mefford HC, et al. 2010a. Exome sequencing identifies MLL2 mutations as a cause of Kabuki syndrome. Nat Genet 42: 790-793. 
Ng SB, Buckingham KJ, Lee C, Bigham AW, Tabor HK, Dent KM, Huff CD, Shannon PT, Jabs EW, Nickerson DA, et al. 2010b. Exome sequencing identifies the cause of a mendelian disorder. Nat Genet 42: 30-35.

Nitta R, Kikkawa M, Okada Y, Hirokawa N. 2004. KIF1A alternately uses two loops to bind microtubules. Science 305: 678-683.

Okada Y, Yamazaki H, Sekine-Aizawa Y, Hirokawa N. 1995. The neuronspecific kinesin superfamily protein KIF1A is a unique monomeric motor for anterograde axonal transport of synaptic vesicle precursors. Cell 81: 769-780.

Otsuka AJ, Jeyaprakash A, Garcia-Anoveros J, Tang LZ, Fisk G, Hartshorne T, Franco R, Born T. 1991. The C. elegans unc-104 gene encodes a putative kinesin heavy chain-like protein. Neuron 6: 113-122.

Pierce SB, Walsh T, Chisholm KM, Lee MK, Thornton AM, Fiumara A, Opitz JM, Levy-Lahad E, Klevit RE, King MC. 2010. Mutations in the DBPdeficiency protein HSD17B4 cause ovarian dysgenesis, hearing loss, and ataxia of Perrault Syndrome. Am J Hum Genet 87: 282-288.

Purcell S, Neale B, Todd-Brown K, Thomas L, Ferreira MA, Bender D, Maller J, Sklar P, de Bakker PI, Daly MJ, et al. 2007. PLINK: a tool set for wholegenome association and population-based linkage analyses. Am J Hum Genet 81: 559-575.

Reid E. 2003. Science in motion: common molecular pathological themes emerge in the hereditary spastic paraplegias. J Med Genet 40: 81-86.

Ropers HH. 2007. New perspectives for the elucidation of genetic disorders. Am J Hum Genet 81: 199-207.

Schwarz JM, Rodelsperger C, Schuelke M, Seelow D. 2010. MutationTaster evaluates disease-causing potential of sequence alterations. Nat Methods 7: 575-576.

Stenson PD, Ball EV, Mort M, Phillips AD, Shiel JA, Thomas NS, Abeysinghe S, Krawczak M, Cooper DN. 2003. Human Gene Mutation Database (HGMD): 2003 update. Hum Mutat 21: 577-581.
Via M, Gignoux C, Burchard EG. 2010. The 1000 Genomes Project: new opportunities for research and social challenges. Genome Med 2: 3. doi $10.1186 / \mathrm{gm} 124$.

Walsh T, Shahin H, Elkan-Miller T, Lee MK, Thornton AM, Roeb W, Abu Rayyan A, Loulus S, Avraham KB, King MC, et al. 2010. Whole exome sequencing and homozygosity mapping identify mutation in the cell polarity protein GPSM2 as the cause of nonsyndromic hearing loss DFNB82. Am J Hum Genet 87: 90-94.

Woods CG, Cox J, Springell K, Hampshire DJ, Mohamed MD, McKibbin M, Stern R, Raymond FL, Sandford R, Malik Sharif S, et al. 2006. Quantification of homozygosity in consanguineous individuals with autosomal recessive disease. Am J Hum Genet 78: 889-896.

Wu X, Jiang R, Zhang MQ, Li S. 2008. Network-based global inference of human disease genes. Mol Syst Biol 4: 189. doi: 1038/msb.2008.27.

Yamada K, Andrews C, Chan WM, McKeown CA, Magli A, de Berardinis T, Loewenstein A, Lazar M, O'Keefe M, Letson R, et al. 2003. Heterozygous mutations of the kinesin KIF21A in congenital fibrosis of the extraocular muscles type 1 (CFEOM1). Nat Genet 35: 318-321.

Yonekawa Y, Harada A, Okada Y, Funakoshi T, Kanai Y, Takei Y, Terada S, Noda T, Hirokawa N. 1998. Defect in synaptic vesicle precursor transport and neuronal cell death in KIF1A motor protein-deficient mice. $J$ Cell Biol 141: 431-441.

Zhao C, Takita J, Tanaka Y, Setou M, Nakagawa T, Takeda S, Yang HW, Terada S, Nakata T, Takei Y, et al. 2001. Charcot-Marie-Tooth disease type 2A caused by mutation in a microtubule motor KIF1Bbeta. Cell 105: 587597.

Received October 27, 2010; accepted in revised form February 28, 2011.

\section{Genome Research}




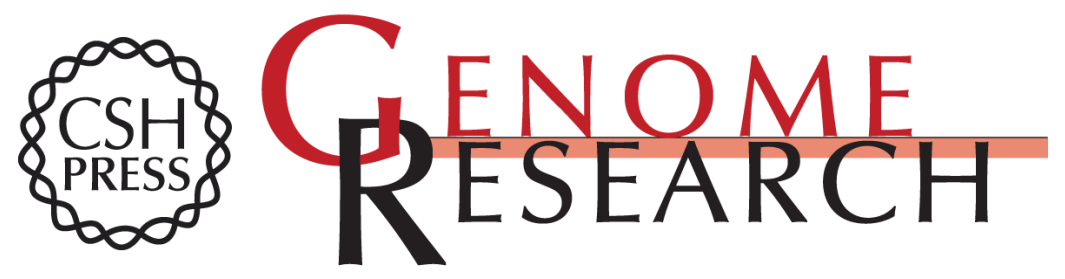

\section{Exome sequencing and disease-network analysis of a single family implicate a mutation in KIF1A in hereditary spastic paraparesis}

Yaniv Erlich, Simon Edvardson, Emily Hodges, et al.

Genome Res. 2011 21: 658-664 originally published online April 12, 2011

Access the most recent version at doi:10.1101/gr.117143.110

\section{Supplemental http://genome.cshlp.org/content/suppl/2011/03/04/gr.117143.110.DC1 Material \\ Related Content Genomic contributions to Mendelian disease \\ Aravinda Chakravarti \\ Genome Res. May , 2011 21: 643-644 Homozygosity mapping and targeted \\ genomic sequencing reveal the gene responsible for cerebellar hypoplasia and quadrupedal locomotion in a consanguineous kindred \\ Suleyman Gulsuner, Ayse Begum Tekinay, Katja Doerschner, et al. \\ Genome Res. December , 2011 21: 1995-2003 Scope note \\ Genome Res. May , 2011 21: xi}

References This article cites 45 articles, 9 of which can be accessed free at:

http://genome.cshlp.org/content/21/5/658.full.html\#ref-list-1

Articles cited in:

http://genome.cshlp.org/content/21/5/658.full.html\#related-urls

\section{License}

Email Alerting Receive free email alerts when new articles cite this article - sign up in the box at the Service top right corner of the article or click here.

\section{Affordable, Accurate Sequencing.}

